\title{
Virtual sensors for active noise control in acoustic-structural coupled enclosures using structural sensing: Robust virtual sensor design
}

\author{
Dunant Halim, Li Cheng, ${ }^{\text {a) }}$ and Zhongqing Su \\ Department of Mechanical Engineering, The Hong Kong Polytechnic University, Kowloon, Hong Kong
}

(Received 10 September 2010; revised 2 December 2010; accepted 3 December 2010)

\begin{abstract}
The work was aimed to develop a robust virtual sensing design methodology for sensing and active control applications of vibro-acoustic systems. The proposed virtual sensor was designed to estimate a broadband acoustic interior sound pressure using structural sensors, with robustness against certain dynamic uncertainties occurring in an acoustic-structural coupled enclosure. A convex combination of Kalman sub-filters was used during the design, accommodating different sets of perturbed dynamic model of the vibro-acoustic enclosure. A minimax optimization problem was set up to determine an optimal convex combination of Kalman sub-filters, ensuring an optimal worst-case virtual sensing performance. The virtual sensing and active noise control performance was numerically investigated on a rectangular panel-cavity system. It was demonstrated that the proposed virtual sensor could accurately estimate the interior sound pressure, particularly the one dominated by cavitycontrolled modes, by using a structural sensor. With such a virtual sensing technique, effective active noise control performance was also obtained even for the worst-case dynamics.
\end{abstract}

(C) 2011 Acoustical Society of America. [DOI: 10.1121/1.3531941]

PACS number(s): 43.55.Jz, 43.60.Bf, 43.40.Rj [NX]

Pages: $1390-1399$

\section{INTRODUCTION}

In the past several decades, there has been considerable work devoted to the active control of low-frequency noise and vibration for vibro-acoustic systems, which can be implemented on systems such as the interior of buildings or aircraft/automotive cabins. Part of the work focused on active control of structural sound radiation to a free field, while other on acoustic-structural coupled enclosures (such as Pan and Hansen, 1991; Snyder and Tanaka, 1993; Cazzolato, 1999; Kim and Brennan, 2000; Tanaka and Kobayashi, 2006; Hill et al., 2009; Li and Cheng, 2010 and references therein). Two active control strategies in this aspect are the global and local active controls, showing respective merits and limitations. The global active control targets the overall noise reduction in a cavity, in terms of acoustic quantities such as acoustic potential energy. In general, the global active control can be successfully achieved at a resonance by targeting the dominant resonances that contribute to the cavity acoustic potential energy in a low modal density region (Bullmore et al., 1987). However, when the response is contributed by a large number of modes such as in the off-resonance region, global control is likely to be less effective (Thomas et al., 1993).

On the other hand, the local active control only reduces interior noise by creating a zone of quiet, which only requires a smaller number of secondary sources compared to that of global active control. Various active control algorithms with fixed or adaptive control filters can be used to minimize signal's energy at error sensors so as to create a zone of quiet inside a cavity. However, the local control

\footnotetext{
a) Author to whom correspondence should be addressed. Electronic mail: mmlcheng@inet.polyu.edu.hk
}

performance is limited due to the localized nature of the zone of quiet. Elliott et al. (1988) studied active pressure cancellation using a remote secondary/control source in a pure-tone diffuse field. It was found that, on average, the shape of zone of quiet with more than $10 \mathrm{~dB}$ pressure reduction was a sphere around the cancellation point with a diameter of only one-tenth of the acoustic wavelength. By controlling pressure and pressure gradient quantities, the 10 $\mathrm{dB}$ zone of quiet can be increased to one-half of the wavelength along the axis of pressure gradient (Elliott and Garcia-Bonito, 1995). Nevertheless, the spatial extent of effective noise reduction is very limited, and moreover, the local active control action may increase the noise outside the zone of quiet so that error sensors must be placed at or very close to the target locations. It is envisaged that it may not be convenient to place an error sensor close to one's ears for active control purposes. This leads to an alternative sensing method, the virtual sensing method, to create a zone of quiet at a target location using a real/physical sensor at some distance away.

A number of virtual sensing algorithms have been proposed by various researchers such as the one reviewed by Moreau et al. (2008). The virtual microphone method, proposed by Elliott and David (1992), was used to predict a sound pressure at a distance away from a microphone with the assumption of equal primary sound pressures at locations of the physical and virtual microphones. Popovich (1997) and Roure and Albarrazin (1999) utilized the concept of the remote microphone technique using a set of transfer functions without the need of equal primary sound field assumptions for the physical and virtual microphones. Cazzolato (1999) proposed the forward difference prediction technique based on a polynomial extrapolation of acoustic signals observed at a number of microphones, which did not require 
an a priori system identification of the acoustic system. This method was later extended by using the adaptive leastmean-square (LMS) virtual technique (Cazzolato, 2002) in which an adaptive LMS algorithm was used to obtain the optimal microphone weights for the extrapolation. Based on the optimal state estimation, Petersen et al. (2008) utilized a Kalman filter to design a virtual sensor for active noise control system. There was also virtual sensing work conducted for a diffused sound field system (Moreau et al., 2009) or for moving virtual sensing (Petersen et al., 2007).

All these studies focused on using acoustic sensors, such as microphones or energy density sensors, to predict acoustic quantities at virtual locations. They were not intended to be used for vibro-acoustic applications, in which sensors in a structural domain were used for virtual sensing in an acoustic domain. However, for vibro-acoustic applications, it can be useful to avoid the use of potentially bulky acoustic sensors and use compact structural vibration sensors instead, such as those used in active structural acoustic control (ASAC). Furthermore, most of the work did not specifically consider the robustness issue, although Kalman-based virtual sensors (Petersen et al., 2008) have some inherent robustness to process and measurement noises. Other works such as that conducted by Wenzel et al. (2007) also utilized Kalman filter as a virtual sensor although for non-acoustic automotive control applications. The majority of the work in robust control using virtual sensors was dedicated to diffracted sound field for active headrest applications (e.g., Rafaely and Elliot, 1999; Pawelczyk, 2003) rather than for vibro-acoustic systems. The work concerned the design of robust control algorithms, such as using $\mathscr{H}_{2} / \mathscr{H}_{\infty}$ control method, to accommodate possible changes in system dynamics and not particularly on the robustness of the virtual sensing algorithm itself.

Still, there are challenges for developing the vibro-acoustic virtual sensors. First, the interior acoustic responses can only be sensed structurally in terms of the fluid-structural coupling. Such a coupling is usually weak due to the relatively high stiffness of the panel and the low density of air (Pan and Bies, 1990; Cheng, 1994). This concern is articulated when an acoustic excitation is considered because the system responses are dominated by acoustic modes that need to be detected accurately using structural sensors. Second, structural measurements of a weakly coupled vibro-acoustic system are very sensitive to changes in system dynamic and structural measurement noise. Hence, the virtual sensing robustness can be critical since the virtual sensor accuracy may be significantly degraded, negatively impacting on the active control performance. To achieve a guaranteed virtual sensing performance over possible uncertain systems, a virtual sensor needs to be designed with an explicit consideration of sensing robustness. Considering the above challenges in the existing techniques, the present work targets at a robust virtual sensing design methodology for sensing and active control applications of vibro-acoustic systems. The contributions of our work are twofold. First, the work proposed a virtual sensor for vibroacoustic applications, using only structural sensors to estimate the interior sound pressures of an acoustic-structural coupled enclosure. Second, the work investigated the issue of virtual sensing robustness by developing a robust virtual sensor design methodology which took into account possible dynamic uncertainties in the system.

The structure of the paper is as follows: Sec. II discusses the uncertain dynamic model of an acoustic-structural coupled enclosure which is used to develop a robust virtual sensor. Section III proposes a robust virtual sensor methodology which takes into account possible dynamic uncertainties in the vibro-acoustic system and measurement noise of structural sensors. Section IV provides a numerical analysis on the virtual sensor design, virtual sensing, and active noise control performances on a rectangular panel-cavity system. The conclusion of the work is presented in Sec. V.

\section{DYNAMIC MODEL OF PERTURBED VIBRO-ACOUSTIC SYSTEMS}

As this work proposed a method for designing virtual sensors without the use of acoustic sensors, the consideration of fluid-structural interactions becomes important. A rectangular coupled panel-cavity system as shown in Fig. 1 was chosen as a representative of vibro-acoustic systems, whose dynamic behavior can be described using the modal-coupling method (Fahy, 1985) from the governing partial differential equations:

$$
\nabla^{2} p(x, y, z, t)-\frac{1}{c^{2}} \ddot{p}(x, y, z, t)=-\rho \dot{q}+2 \rho \ddot{w}(x, y, t) \delta\left(z-z_{0}\right),
$$

$$
\begin{aligned}
D \nabla^{4} w(x, y, t)+m \ddot{w}(x, y, t)= & f(t) \delta\left(x-x_{f}\right) \delta\left(y-y_{f}\right) \\
& +p\left(x, y, z_{0}, t\right)
\end{aligned}
$$

where $p, q, w, c, \rho, m$, and $f$ are the sound pressure in the cavity at location $(x, y, z)$, acoustic source volume velocity per unit volume, panel displacement at location $(x, y)$, speed of sound in the fluid, fluid density, panel mass per unit area, and the external point force applied at location $\left(x_{f}, y_{f}\right)$,

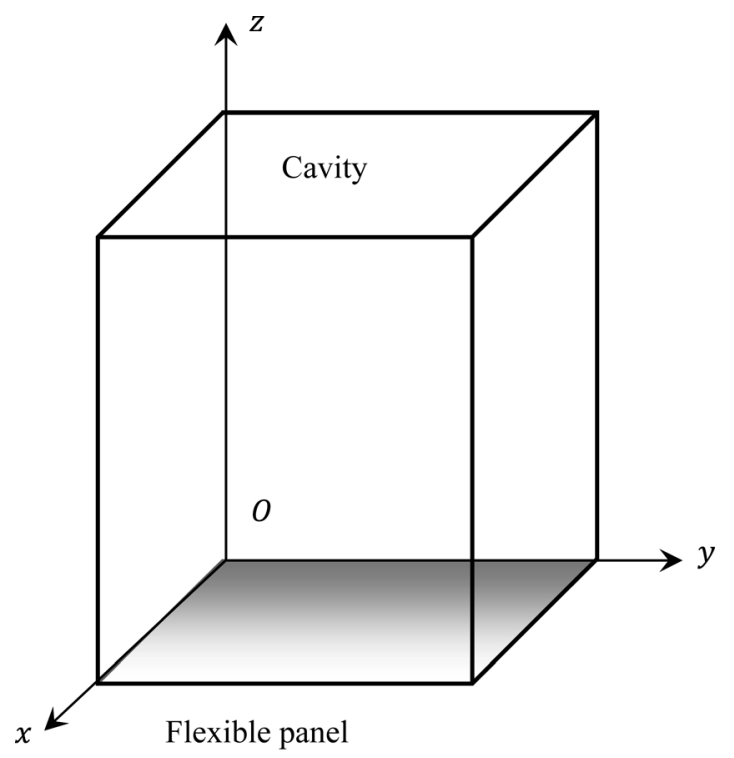

FIG. 1. A rectangular acoustic-structural coupled system used for numerical validation. The flexible panel is located at the bottom of the cavity. 


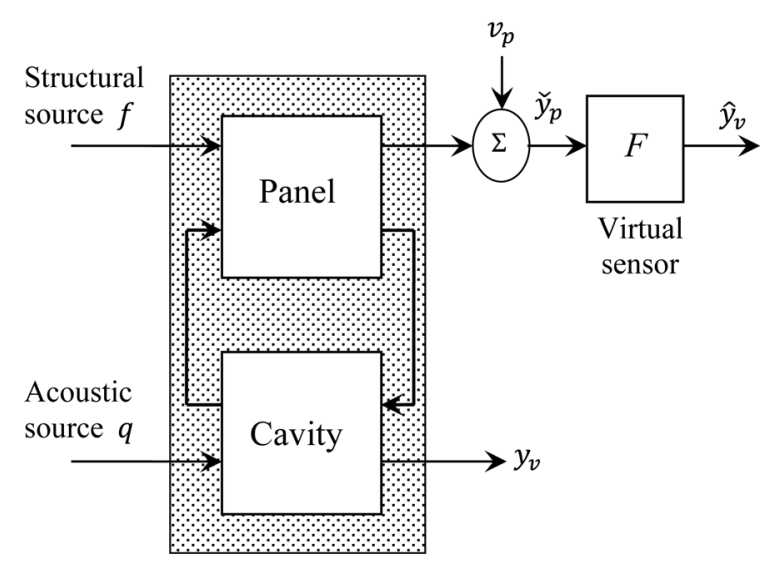

FIG. 2. A generic virtual sensor configuration for a coupled panel-cavity system with structural and acoustic excitations. The virtual sensor filter $F$ utilized information from the structural measurement to estimate the interior sound pressure. For clarity, deterministic excitations were not shown.

respectively. The panel stiffness, $D$, is obtained from $D=E d^{3} / 12\left(1-v^{2}\right)$ where $E, d$, and $v$ are Young's modulus, thickness, and Poisson's ratio of the panel, respectively. In addition, $\delta(\cdot)$ is the Dirac delta function and $z_{0}$ is the location of a flat panel in $z$-coordinate. It should be noted that this work is also applicable for more complex systems whose modal properties can be obtained from numerical modeling or experimental methods.

The modal decomposition method (Fahy, 1985) is used by representing the structural and acoustic responses with their respective in-vacuo panel and rigid-walled cavity eigenfunctions as well as their time-dependent modal displacement and sound pressure. The state space model can then be constructed from the set of ordinary differential equations.

Figure 2 depicts the general configuration of the proposed virtual sensing design. Deterministic excitations were not shown in the figure for the sake of clarity. There are two general types of disturbance sources, structural $f$ and acoustic $q$, that may excite the vibro-acoustic system. In designing a virtual sensor for such a system, the main aim is to robustly estimate the acoustic pressure, $y_{v} \in \mathbb{R}^{N_{v}}$ at $N_{v}$ cavity locations, using the information obtained from $N_{p}$ structural sensors, $\check{y}_{p} \in \mathbb{R}^{N_{p}}$, whose measurements are corrupted by measurement noise, $v_{p} \in \mathbb{R}^{N_{p}}$.

A linear time-invariant state space model of a vibroacoustic system can be described by

$$
\begin{aligned}
& \dot{\sigma}(t)=A \sigma(t)+B_{d} d(t)+B_{u} u(t), \\
& \check{y}_{p}(t)=C_{p} \sigma(t)+v_{p}(t), \\
& y_{v}(t)=C_{v} \sigma(t),
\end{aligned}
$$

where $\sigma \in \mathbb{R}^{N_{\sigma}}, d \in \mathbb{R}^{N_{d}}$, and $u \in \mathbb{R}^{N_{u}}$ are the $N_{\sigma}$ states of the plant, $N_{d}$ acoustic or structural process/disturbance inputs, and $N_{u}$ control inputs, respectively. Furthermore, $A \in \mathbb{R}^{N_{\sigma} \times N_{\sigma}}, B_{d} \in \mathbb{R}^{N_{\sigma} \times N_{d}}, B_{u} \in \mathbb{R}^{N_{\sigma} \times N_{u}}, C_{p} \in \mathbb{R}^{N_{p} \times N_{\sigma}}$, and $C_{v} \in \mathbb{R}^{N_{v} \times N_{\sigma}}$, respectively, denote the state matrix, input matrices for disturbance and control sources, and the output matrices for structural sources and acoustic sensors at virtual locations. Note that in this study for virtual sensing purposes, we are interested in the acoustic pressure response $y_{v}$ which was directly correlated to disturbance inputs. This explains why an uncorrelated noise term was not included in $y_{v}$ expression in Eq. (5) since it cannot be "observed" by structural sensors.

In contrast to a more conservative norm-bounded uncertainty model, the polytope parameter uncertainty model with convex bounded uncertainties (Lewis et al., 2008) was used to represent the possible variations of dynamic model of a vibro-acoustic system. The model can provide descriptions of parameter uncertainties that may occur in actual physical systems, such as variations in resonant frequencies or damping. Consider a perturbed vibro-acoustic system from input $\left[d^{T} u^{T} v_{p}^{T}\right]^{T}$ to output $\left[\check{y}_{p}{ }^{T} y_{v}{ }^{T}\right]^{T}$

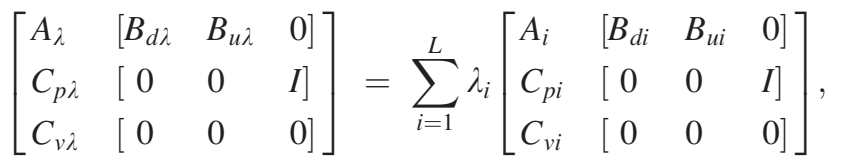

where subscripts $\lambda$ and $i$, respectively, denote the state space matrices associated with the polytope model $\lambda$ and the $i$ th vertex model. Here, $L$ is the number of vertices from the polytopic parameter uncertainty model and the polytope parameter is denoted by $\lambda$ which belongs to a unit simplex

$$
\Lambda=\left\{\lambda \in \mathbb{R}^{\mathrm{L}}, \sum_{i=1}^{\mathrm{L}} \lambda_{i}=1, \lambda_{i} \geq 0\right\} .
$$

\section{ROBUST VIRTUAL SENSOR DESIGN}

It is well known that the Kalman filter is a linear optimal filter, in a mean-square sense, for a dynamic system under stochastic process and measurement noises (Gelb, 1974). Note that the optimal continuous filter is called KalmanBucy filter, but it is referred to as the Kalman filter hereinafter in the study for brevity. Such a Kalman filter has been used for designing a virtual sensor for purely acoustic systems (Petersen et al., 2008). In contrast, our work focused on developing vibro-acoustic virtual sensors for a system with certain dynamic uncertainties.

For an uncertain dynamic model, let $\bar{\lambda}_{j}$ $=\left[\lambda_{j 1}, \ldots, \lambda_{j L}\right]^{T} \in \Lambda$ be a set of $\lambda_{i}$ values representing a particular polytope model inside a convex set $\Lambda$, not necessarily at the vertices. This uncertain dynamic model $\bar{\lambda}_{j}$ can be represented by $G_{p d}\left(\omega, \bar{\lambda}_{j}\right) \triangleq\left(A_{j}, B_{d j}, C_{p j}, 0\right)$ and $G_{v d}\left(\omega, \bar{\lambda}_{j}\right) \triangleq\left(A_{j}, B_{d j}, C_{v j}, 0\right)$, which are the transfer functions from the process/disturbance input $d$ to the uncorrupted structural sensor output $y_{p}$ and the acoustic virtual sensor output $y_{v}$, respectively.

Consider the case where the process and measurement noises are white, zero mean, Gaussian, and uncorrelated. The diagonal spectral density matrices for the process and measurement noises are described by $Q_{d}$ and $Q_{n}$, respectively. In this work, multiple Kalman sub-filters $F_{j}(\omega)$ with $j=1, \ldots, L$ were utilized for the virtual sensor design. Consider a nominal model described by polytope parameter $\bar{\lambda}_{j}, G_{p d}\left(\omega, \bar{\lambda}_{j}\right)$, and $G_{v d}\left(\omega, \bar{\lambda}_{j}\right)$. The $j$ th Kalman sub-filter $F_{j}(\omega)$, as an $\mathscr{H}_{2}$-optimal 
linear estimator, minimizes the $\mathscr{H}_{2}$ norm of the virtual sensing error system for the nominal model $G_{d}\left(\omega, \bar{\lambda}_{j}\right)$

$$
\begin{aligned}
& \min _{F_{j} \in \mathcal{F}} J\left(F_{j}(\omega), G_{d}\left(\omega, \bar{\lambda}_{j}\right), Q_{d}, Q_{n}\right) \\
& =\min _{F_{j} \in \mathcal{F}} \|\left[G_{v d}\left(\omega, \bar{\lambda}_{j}\right) \sqrt{Q_{d}} \quad 0\right] \\
& -F_{j}(\omega)\left[G_{p d}\left(\omega, \bar{\lambda}_{j}\right) \sqrt{Q_{d}} \quad \sqrt{Q_{n}}\right] \|_{2}^{2},
\end{aligned}
$$

where $\mathscr{F}$ is the set of all causal and stable linear time-invariant filter and

$$
G_{d}=\left[\begin{array}{c}
G_{v d} \\
G_{p d}
\end{array}\right] .
$$

The steady-state Kalman filter solution is obtained by finding the limiting solution of the error covariance $P_{j}$ associated with the following algebraic Riccati equation under certain convergence conditions (Gelb, 1974; Lewis et al., 2008)

$$
\dot{P}_{j}=A_{j} P_{j}+P_{j} A_{j}^{T}+B_{d j} Q_{d} B_{d j}^{T}-P_{j} C_{p j}^{T} Q_{n}^{-1} C_{p j} P_{j}=0 .
$$

The Kalman gain can then be obtained as

$$
K_{p j}=P_{j} C_{p j}^{T} Q_{n}^{-1}
$$

Although such a Kalman-based virtual sensor can perform well under a nominal system, there is no guarantee that it would perform reasonably well when the system dynamic is no longer at the nominal case. The Kalman filter can be designed for a better sensing performance, though it is still limited for the nominal system. It should be noted that obtaining a virtual sensor filter based on the direct inversion of transfer function associated with structural sensor output, $G_{p d}$, is generally not a practical solution because of potential problems with the stability, causality, and noise sensitivity of such a filter. Transfer function $G_{p d}$ is generally not a collocated system with respect to the disturbance input, i.e., it is a non-minimum phase system which has an unstable inversion. Further, since a vibro-acoustic dynamic model always contains inaccuracies particularly at anti-resonance regions where the signal-to-noise is low, a direct inversion of the model may lead to a virtual sensor filter with high noise sensitivity due to its excessive sensor gain. In this case, the Kalman filter can provide a practical alternative to designing a virtual sensor, although a consideration of robustness in the design is necessary.

In the work, a robust virtual sensor filter was constructed from a convex combination of Kalman sub-filters based on the work proposed by Geromel and Martins (2009). As an improvement, the proposed robust filter design did not use the set of extreme Kalman sub-filters associated with $L$ vertices of the polytope model. A convex combination of Kalman sub-filters was chosen to minimize the performance criterion similar to Eq. (8) but over a range of possible dynamic uncertainties. Each sub-filter represents an optimal Kalman filter that minimizes the virtual sensing error energy for a system described by a certain polytope model as in Eq. (6). The robust virtual sensor is expressed as

$$
F(\omega)=\sum_{j=1}^{L} \kappa_{j} F_{j}(\omega),
$$

where $\kappa \in \Gamma$ with $\Gamma=\left\{\kappa \in \mathbb{R}^{L}, \sum_{i=1}^{L} \kappa_{i}=1, \kappa_{i} \geq 0\right\}$, describes the relative contribution of each Kalman sub-filter, $F_{j}(\omega)$, which needs to be optimized for obtaining a satisfactory robust sensing performance. Note that in general it is possible to set $L$ to be any positive integer number. Since the aim in this study is to develop a practicable approach for virtual sensing of vibro-acoustic system, we limit the design using $L$ as the number of vertices of the polytopic uncertainty model for a tractable optimal filter solution. Figure 3 shows the diagram for the robust vibro-acoustic virtual sensor, with deterministic excitation $u$ included, in which the estimated sound pressure at virtual sensor locations can be minimized using active control algorithms such as the adaptive filtering ones. Multiple Kalman sub-filters, as shown in Fig. 3(b), generated partial estimates for the interior sound pressure, which were summed up to produce an estimate of sound pressure at virtual locations.

The robust virtual sensor filter was designed by minimizing the worst virtual sensing error subject to broadband disturbance $\left[d^{T} v_{p}^{T}\right]^{T}$. The optimal solution for $\kappa_{j}$ 's $(j=1, \ldots, L)$ was obtained by setting up a constrained minimax optimization

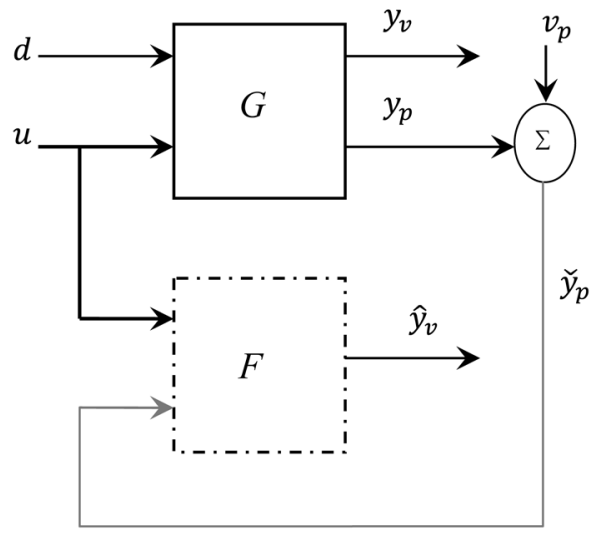

(a)

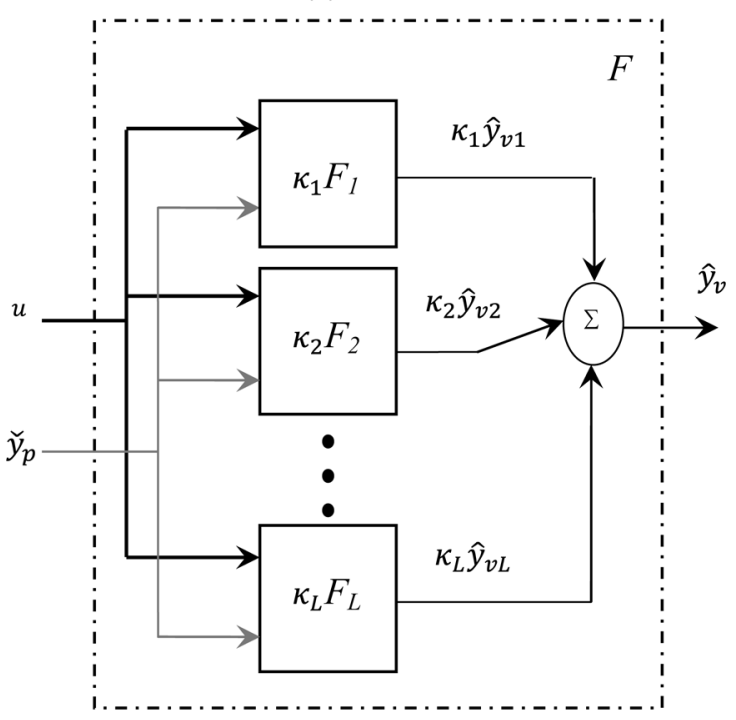

(b)

FIG. 3. The vibro-acoustic robust virtual sensor diagram. (a) A general diagram showing the virtual sensor filter $F$. (b) The composition of the virtual sensor $F$ as a convex combination of multiple Kalman sub-filters. 


$$
\begin{array}{r}
\min _{\kappa \in \Gamma} \max _{\lambda \in \Lambda}\left\|E_{v}(\omega, \lambda)\right\|_{2}^{2} \\
=\min _{\kappa \in \Gamma} \max _{\lambda \in \Lambda} \| \sum_{j=1}^{L} \kappa_{j}\left[\left(G_{v d}(\omega, \lambda)-F_{j}(\omega) G_{p d}(\omega, \lambda)\right) \sqrt{Q_{d}}\right. \\
\left.-F_{j}(\omega) \sqrt{Q_{n}}\right] \|_{2}^{2}
\end{array}
$$

where $E_{v}$ is the transfer function from $\left[d^{T} v_{p}^{T}\right]^{T}$ to $e_{v}=y_{v}-\widehat{y}_{v}$ with $\widehat{y}_{v}$ being the estimated sound pressure at virtual sensor locations.

In practice, the bandwidth of virtual sensor is limited usually to low-frequency active control applications only. For this purpose, the model used for virtual sensor design can be constructed by including only relevant low-frequency modes. Further, without loss of generality, colored process and measurement noises can be accommodated such as using a spectrum shaping filter and state augmentation process (Lewis et al., 2008). The direct minimax optimization using a sequential quadratic programming procedure was employed in this study to solve the robust filtering problem for vibro-acoustic systems with a high number of modes.

The state space realization of the virtual sensing error system $E_{v}$ can be constructed in terms of parameter uncertainty $\lambda$, and the optimization problem can be solved. The proposed robust virtual sensor can then be expressed by including the deterministic control input term with the associated input matrix $B_{u j}$

$$
\begin{aligned}
& \frac{\partial \hat{\sigma}_{j}(t)}{\partial t}=A_{k j} \widehat{\sigma}_{j}(t)+K_{p j} \check{y}_{p}(t)+B_{u j} u(t), \quad j=1, \ldots, L, \\
& \hat{y}_{v}(t)=\sum_{j=1}^{L} \kappa_{j} C_{v j} \hat{\sigma}_{j}(t),
\end{aligned}
$$

where $\hat{\sigma}_{j}$ denotes the estimated state vector and $A_{k j}=A_{j}$ $-K_{p j} C_{p j}$.

The proposed robust virtual sensor thus utilizes the structural sensor measurement, corrupted by measurement noise, to predict the states of the vibro-acoustic system. From the estimated states, the desired interior sound pressure at virtual locations in the cavity can be estimated. Each Kalman sub-filter gain $K_{p j}$ depends on the error covariance $P_{j}$ in Eq. (9), which measures the uncertainty level of state estimation, thus affecting the virtual sensing estimation. If the state estimation is less "certain" due to an inaccurate model, the Kalman gain will be "larger" to prioritize the measurements from structural sensors. On the other hand, if the model is relatively accurate, or the measurement noise level is significant, the Kalman gain will be "small" to prioritize the estimation based on the vibro-acoustic model.

In this work, it is generally easier to construct a model with physical insight in continuous time rather than in discrete time (Ljung, 1999). For the virtual sensor implementation, the optimized virtual sensor filter can then be discretized before it is implemented on the vibro-acoustic system. For a small sampling time, which is normally used for sensing and active control applications of vibro-acoustic systems, there is a direct relationship between discrete and continuous Kalman filter structures (Lewis., 2008), which allows a simple conversion between discrete and continuous robust virtual sensor filters for virtual sensing implementations.

\section{NUMERICAL VALIDATION OF ROBUST VIRTUAL SENSOR DESIGN}

To validate the proposed optimal virtual sensor design methodology, a rectangular coupled panel-cavity system with dimensions of $(0.695 \mathrm{~m}, 0.976 \mathrm{~m}, 1.188 \mathrm{~m})$ was utilized as a benchmark. One side of the cavity was covered by an aluminum panel of size $(0.695 \mathrm{~m}, 0.976 \mathrm{~m})$ and thickness of $4 \mathrm{~mm}$ at $z_{0}=0$, as depicted in Fig. 1. The proportional damping ratios for the in-vacuo panel and rigid-walled cavity modes were assumed to be 0.005 and 0.003 , respectively. The highly resonant system used in this study served the purpose of demonstrating the robust virtual sensing effectiveness. The natural frequencies of the coupled panel-cavity modes are shown in Table I with the frequency range of interest up to around 300 $\mathrm{Hz}$ containing of the first 20 natural frequencies.

The acoustic virtual sensor inside the cavity was located at the coordinate $\left(x_{c}, y_{c}, z_{c}\right)=(0.070 \mathrm{~m}, 0.816 \mathrm{~m}, 1.028 \mathrm{~m})$ and a structural velocity sensor at $(0.297 \mathrm{~m}, 0.360 \mathrm{~m})$ was used for virtual sensor design, whose placement was a priori determined in Halim et al. (2010). The design criterion of the sensor location was to ensure that the structural sensor output energy was dominated by the modes that were important for the virtual sensor. For this velocity sensor location, only dominant first, seventh, and ninth panel-controlled modes and dominant second-sixth cavity-controlled modes were targeted, as those modes were relevant for this particular virtual sensor location. In this analysis, the system eigenfunctions were unchanged although the natural frequencies

\begin{tabular}{|c|c|}
\hline Mode number and $n$ or $h$ & Frequency $(\mathrm{Hz})$ \\
\hline $1[h=1,(0,0,0)]$ & 0.0 \\
\hline $2[n=1,(1,1)]$ & 31.6 \\
\hline $3[n=2,(1,2)]$ & 59.8 \\
\hline $4[n=3,(2,1)]$ & 89.2 \\
\hline $5[n=4,(1,3)]$ & 110.3 \\
\hline $6[n=5,(2,2)]$ & 119.6 \\
\hline $7[h=\mathbf{2},(\mathbf{0}, \mathbf{0}, \mathbf{1})]$ & 145.9 \\
\hline $8[n=6,(2,3)]$ & 169.9 \\
\hline $9[h=3,(0,1,0)]$ & 175.9 \\
\hline $10[n=7,(1,4)]$ & 181.6 \\
\hline $11[n=8,(3,1)]$ & 188.8 \\
\hline $12[n=9,(3,2)]$ & 218.4 \\
\hline $13[h=4,(0,1,1)]$ & 229.7 \\
\hline $14[n=10,(2,4)]$ & 240.3 \\
\hline $15[h=5,(1,0,0)]$ & 248.1 \\
\hline $16[n=11,(3,3)]$ & 269.1 \\
\hline $17[n=12,(1,5)]$ & 271.3 \\
\hline $18[h=6,(1,0,1)]$ & 287.4 \\
\hline $19[h=7,(0,0,2)]$ & 290.7 \\
\hline $20[h=8,(1,1,0)]$ & 304.5 \\
\hline
\end{tabular}
and modal damping were perturbed. Note that in a more

TABLE I. First 20 natural frequencies of a coupled panel-cavity system [ $n$ or $h$ indicates the $n$th panel-controlled or $h$ th cavity-controlled modes (shown in bold), respectively]. 
general case where the eigen-functions are expected to change significantly, variations in modal observability levels can be considered for optimizing the sensor placement.

In this validation, the task was to design a robust virtual sensor filter that performed reasonably well in the case of certain dynamic uncertainties in the system. The natural frequencies of the system were assumed to vary by $\pm 1.5 \%$, with the damping being proportionally affected. This variation amounted to resonant frequency variations up to $\pm 4.3 \mathrm{~Hz}$ for the sixth cavitycontrolled resonance. The uncertainty in natural frequencies of the modes was used as a representative problem to demonstrate the virtual sensor's robust performance. The vibro-acoustic system dynamics was allowed to be time-variant within a certain perturbation range, while the time-invariant virtual sensor had to still accurately sense the interior sound pressure. This is a useful practical application where the dynamics of vibro-acoustic systems, such as the natural frequencies and damping, are not known accurately and may change during the operations.

An acoustic volume velocity source at $(0.430 \mathrm{~m}, 0.330 \mathrm{~m}$, $0.850 \mathrm{~m}$ ) inside the cavity was used as the primary disturbance source. Here, an acoustic disturbance was used to emphasize the virtual sensor performance in detecting cavity-controlled modes by using structural sensors only. This is a more challenging problem than if the disturbance source is structural. In the case of structural disturbance, vibro-acoustic modes that are strongly dominated by structural vibration would be excited, and such structural vibration can be directly detected by structural sensors. In contrast, the cavity-controlled acoustic modes are generally weakly excited by the structural disturbance due to the weak structural-acoustic coupling. On the other hand, an acoustic disturbance can strongly excite cavitycontrolled acoustic modes that however cannot be sensed directly by structural sensors except by considering the structural-acoustic coupling. In practical applications, acoustic disturbance can occur and this virtual sensor design avoids using acoustic sensors and instead relying on structural sensors that can be compactly embedded on to existing flexible panels.

The vibro-acoustic system was modeled using a polytopic parameter uncertainty model in Eq. (6) with two vertices $L=2$, representing the natural frequency uncertainties of $+1.5 \%$ for $\left(\lambda_{1}=0.0, \lambda_{2}=1.0-\lambda_{1}=1.0\right)$ and $-1.5 \%$ for $\left(\lambda_{1}=1.0, \lambda_{2}=1.0-\lambda_{1}=0.0\right)$. The strength of process/disturbance and measurement noises was described by discrete covariances of $10^{-10} \mathrm{~m}^{6} / \mathrm{s}^{2}$ and $10^{-14} \mathrm{~m}^{4} / \mathrm{s}^{2}$, respectively, in which a sampling frequency of $10 \mathrm{KHz}$ was used.

In designing a robust filter, the use of "extreme" Kalman sub-filters, as proposed by Geromel and Martins (2009), may not always achieve the best robust performance. In other words, choosing candidates of Kalman sub-filters based on nominal models with the extreme parameter variations may not be the best option. In this work, the minimum error variance (best sensing) and maximum error variance (worst sensing) of a virtual sensor filter were investigated as functions of polytope parameters of two nominal models, $\bar{\lambda}_{1}$ and $\bar{\lambda}_{2}$. Two Kalman sub-filters were chosen to achieve a relatively small maximum error variance for robust performance, while avoiding a substantial increase of its minimum error variance. The selected two nominal models were described by $\bar{\lambda}_{1}=(0.67,0.33)$ and $\bar{\lambda}_{2}=(0.33,0.67)$. The optimal solution for the associated robust virtual sensor filter is $\left[\kappa_{1}, \kappa_{2}\right]^{T}=[0.673,0.327]^{T}$ with the filter's normalized minimum and maximum error variances of 0.055 and 0.330 , respectively, as can be seen from the solid line in Fig. 5.

\section{A. Virtual sensing performance}

The magnitudes of nominal frequency responses from the disturbance input to the acoustic virtual and structural vibration outputs are shown in Figs. 4(a) and 4(b), respectively. At the acoustic virtual sensor output, the second-sixth cavitycontrolled modes (indicated by $\odot$ ) are clearly observable as shown by high resonance responses. Figure 4(b) shows that the structural sensor output had relatively high responses at the resonances of modes of interest, particularly for the first and seventh panel-controlled modes and the second-fourth cavity-controlled modes. The robust virtual sensor response is shown in Fig. 4(c). The result shows that the filter gain was generally higher when the structural sensor output was low, indicating the importance of structural sensor placement.

Figure 5 depicts the sensing error variance, normalized by the variance of $y_{v}$, for the robust virtual sensor and standard nominal Kalman virtual sensor. Here, $\lambda_{1}$ is the polytope

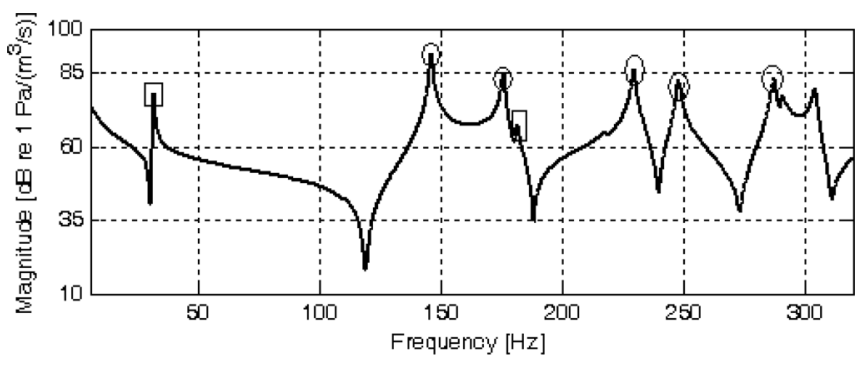

(a)

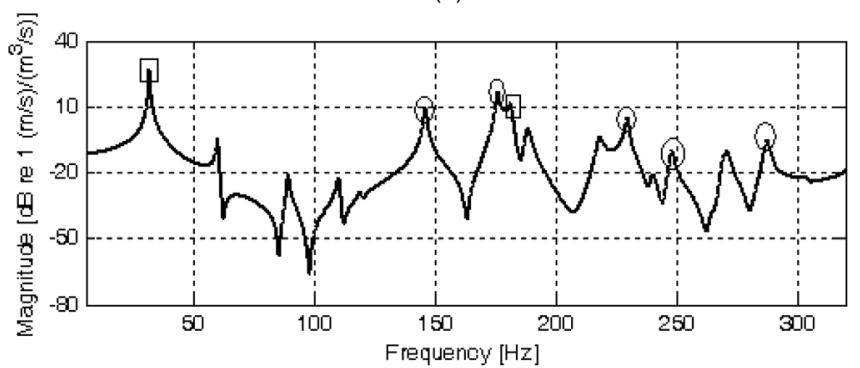

(b)

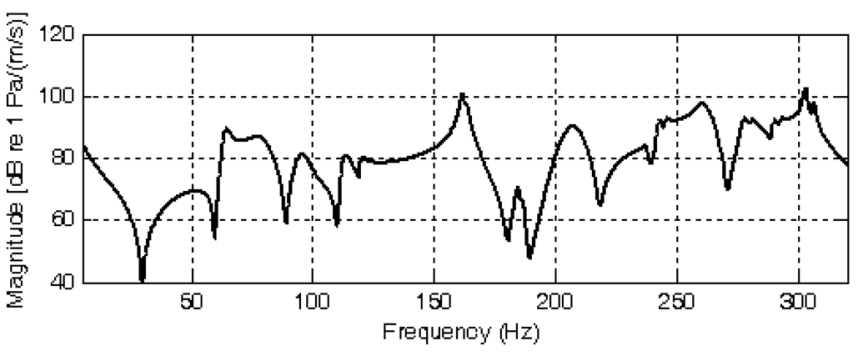

(c)

FIG. 4. The magnitude of nominal frequency responses from an acoustic volume velocity source at $(0.430 \mathrm{~m}, 0.330 \mathrm{~m}, 0.850 \mathrm{~m})$ to: (a) acoustic pressure at virtual sensing location $(0.070 \mathrm{~m}, 0.816 \mathrm{~m}, 1.028 \mathrm{~m})$ and (b) structural velocity at $(0.297 \mathrm{~m}, 0.360 \mathrm{~m})$. $\square$, first and seventh panel-controlled modes; $\odot$, second-sixth cavity-controlled modes. (c) Magnitude of frequency response for the robust virtual sensor. 


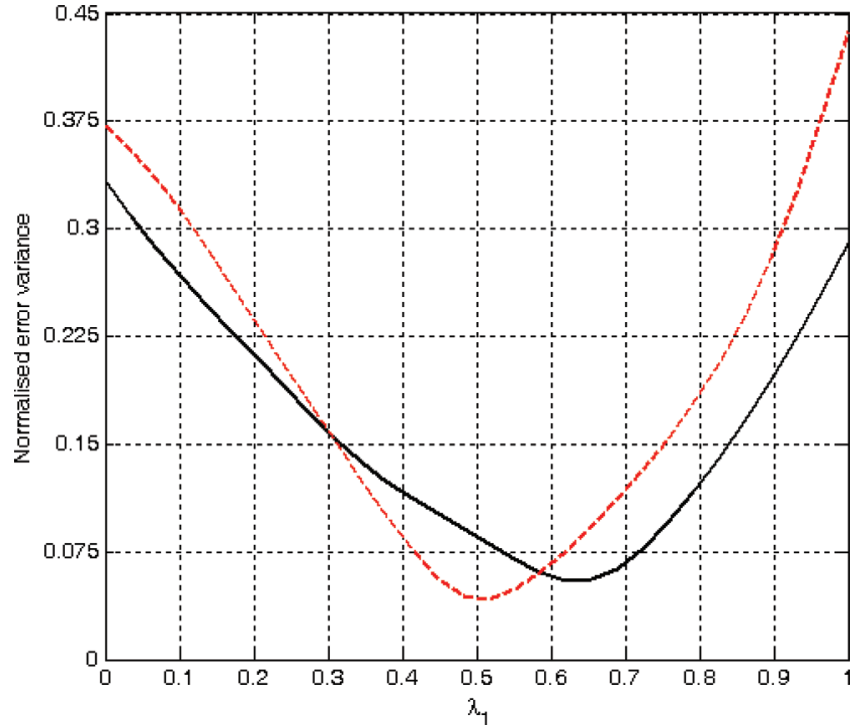

FIG. 5. (Color online) Normalized virtual sensing error variances for varying system uncertainties denoted by $\lambda_{1}$ for the standard Kalman filter (dashed line) and robust virtual (solid line) sensors using a structural velocity sensor at $(0.297 \mathrm{~m}, 0.360 \mathrm{~m})$.

parameter in Eq. (7), whose values of 0.0, 0.5, and 1.0 representing resonant frequency uncertainties of $+1.5 \%, 0 \%$ (nominal), and $-1.5 \%$, respectively. The performance of the robust virtual sensor is shown by the solid line for varying system uncertainties. The sensing performance of a nominal Kalman filter with the same structural sensor is shown by the dashed line. This filter expectedly had the lowest variance for a nominal system at $\lambda_{1}=0.5$ but an approximately $50 \%$ worse variance at $\lambda_{1}=1.0$ than that of the robust virtual sensor. The proposed robust virtual sensor performed better at most of the system uncertainties as intended.

Figures 6(a)-6(c) illustrate the broadband sensing performance of the robust virtual sensor at the worst-case system. A broadband volume velocity disturbance with a bandwidth of $320 \mathrm{~Hz}$ was applied to the system, as shown in Fig. 6(a), whose structural velocity vibration was observed by the structural sensor as shown in Fig. 6(b). Figure 6(c) shows the comparison of the true and estimated sound pressures at the virtual sensor location inside the cavity (solid line for the actual sound pressure; dotted line for the estimated sound pressure). As observed, the virtual sensor was still able to estimate the sound pressure with reasonable accuracy even for the worst case.

Figure 7(a) depicts the frequency domain performance for the nominal system, where cavity sound pressures were accurately estimated over most of frequencies. At the worst case where $\lambda_{1}=0.0$ (the resonance frequencies were perturbed by $+1.5 \%$ ) as shown in Fig. 7(b), only the estimation of the third and eighth cavity-controlled resonances at around 178.5 and $309.0 \mathrm{~Hz}$ suffer. (Note that the eighth cavity-controlled mode was not targeted in the virtual sensor design.) However, the virtual sensing was still reasonably accurate, considering that the virtual sensor was a timeinvariant filter and the system was highly resonant. For comparison, using the quality-factor calculation and assuming a weakly coupled approximation, the $3 \mathrm{~dB}$ bandwidth at (a)

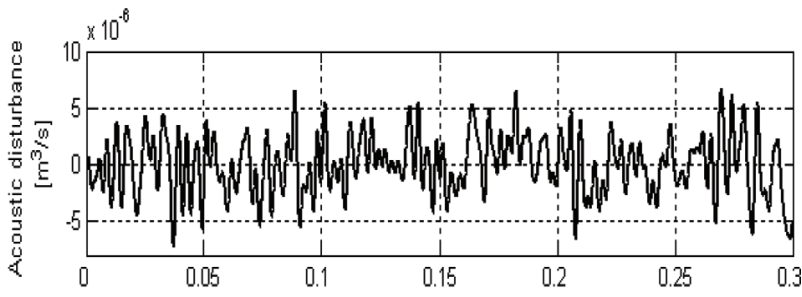

(b)

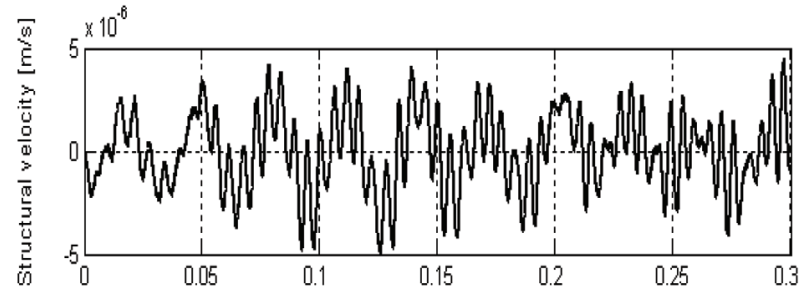

(c)

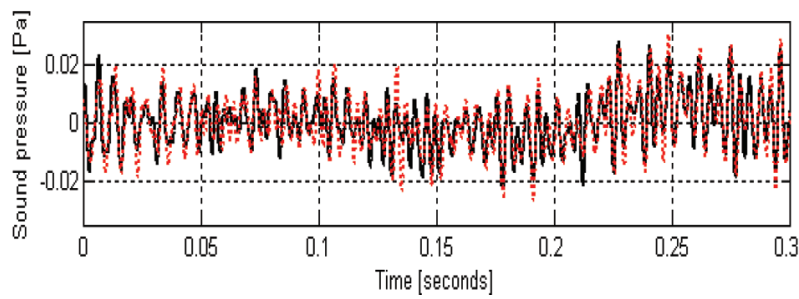

FIG. 6. (Color online) Broadband virtual sensing performance in time-domain. (a) The $320 \mathrm{~Hz}$ bandwidth acoustic disturbance. (b) Measured structural velocity at the structural sensor location. (c) Cavity interior sound pressure at the virtual sensor location: true (solid line) and estimated (dotted line) sound pressures.

resonances was only up to $1.0 \%$, relative to their resonant frequencies. Thus $3.0 \%$ resonant frequency uncertainties used in this numerical study were rather significant, which demonstrate the performance of the proposed virtual sensor.

Furthermore, the proposed virtual sensor consequently has a certain robustness property against variations in disturbance input distribution. When the distribution or location of disturbance input varies, a different proportion of vibro-acoustic modes will be excited. As our virtual sensor is a broadband filter that considers sensing contribution from a number of modes within a frequency band, the sensor can still accurately estimate the sound pressure contributed by a different proportion of these modes. The sensing performance obviously depends on whether these modes are included in the virtual sensor design so that the sensor can be made sensitive to observing these modes. Therefore, one can design the sensor to focus on a number of important vibro-acoustic modes although the disturbance location or distribution may not be accurately known.

\section{B. Active noise control performance using virtual sensors}

Based on the developed virtual sensor, active noise control was used to minimize noise at the virtual location. A volume velocity secondary source was located at $(0.200 \mathrm{~m}$, $0.350 \mathrm{~m}, 0.140 \mathrm{~m}$ ) of system coordinates. The secondary sound pressure frequency responses for the nominal and worst cases are shown in Fig. 8. As expected, due to the deterministic nature of control input $u$, the virtual sensing accuracy was better than that for the primary responses shown in Fig. 7. Based on the principle of active tonal control 


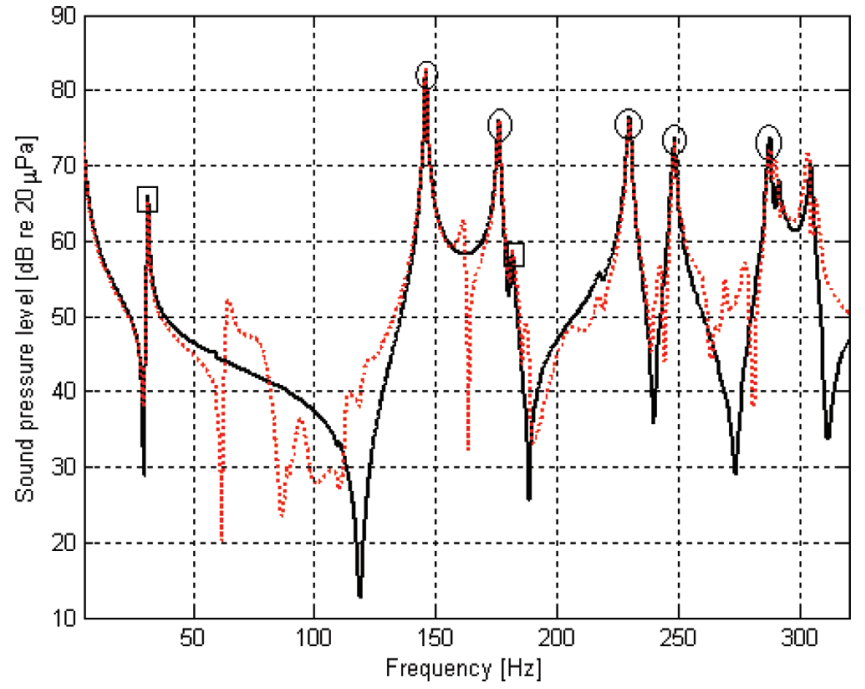

(a)

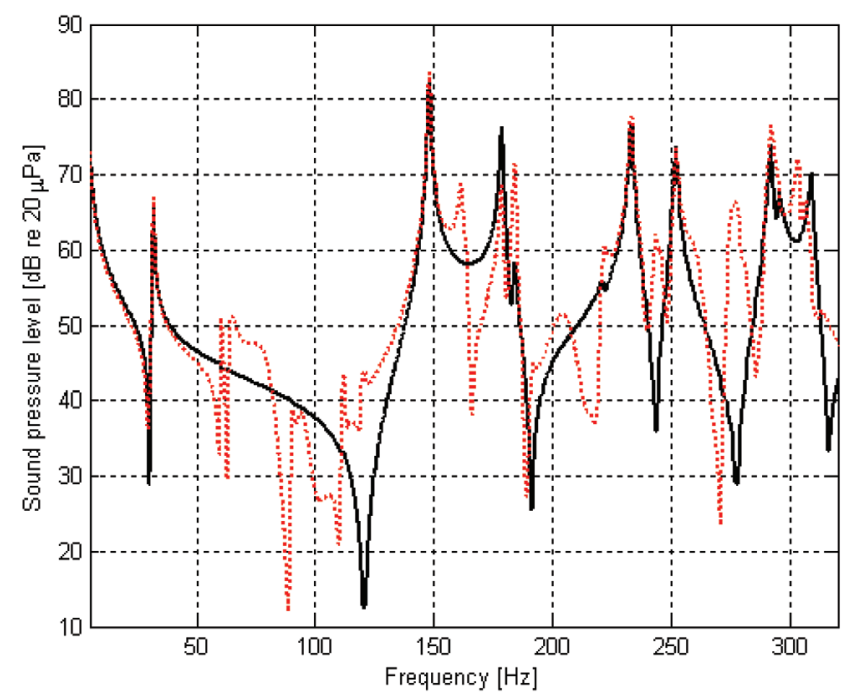

(b)

FIG. 7. (Color online) Primary sound pressure frequency responses due to $10^{-5} \mathrm{~m}^{3} / \mathrm{s}$ root-mean-square (rms) acoustic volume velocity disturbance input at $(0.430 \mathrm{~m}, 0.330 \mathrm{~m}, 0.850 \mathrm{~m})$ for (a) nominal case and (b) worst case. Solid line, the true sound pressure response; dotted line, the estimated sound pressure response from the virtual sensor. $\square$, first and seventh panelcontrolled modes; $\odot$, second-sixth cavity-controlled modes.

(Elliott, 2001), the noise at virtual location can be expressed by assuming the negligible measurement noise $v_{p}$ term

$$
\begin{aligned}
& \hat{y}_{v}(\omega)=\hat{y}_{v d}(\omega)+G_{\hat{v} u}(\omega) u(\omega), \\
& u_{c}(\omega)=-G_{\hat{v} u}^{\dagger}(\omega) \hat{y}_{v d}(\omega), \\
& y_{v}(\omega)=y_{v d}(\omega)-G_{v u}(\omega) G_{\hat{v} u}^{\dagger}(\omega) \hat{y}_{v d}(\omega),
\end{aligned}
$$

where $\hat{y}_{v d}$ and $y_{v d}$ are the estimated and true primary sound pressures at the virtual location, $G_{\hat{v} u}$ is the transfer function from control input to the virtual sound pressure, $u_{c}$ is the optimal control input based on the pseudo-inverse of $G_{\hat{v} u}$, and $G_{v u}$ is the transfer function from control input to the true sound pressure. Note that a high level of measurement noise $v_{p}$ can degrade the virtual sensing accuracy, thus affecting

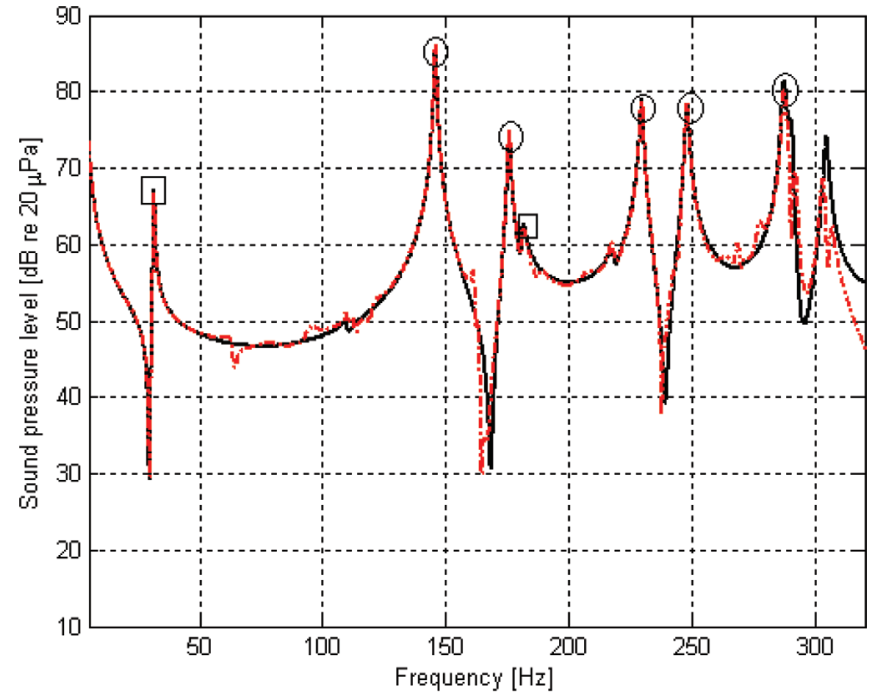

(a)

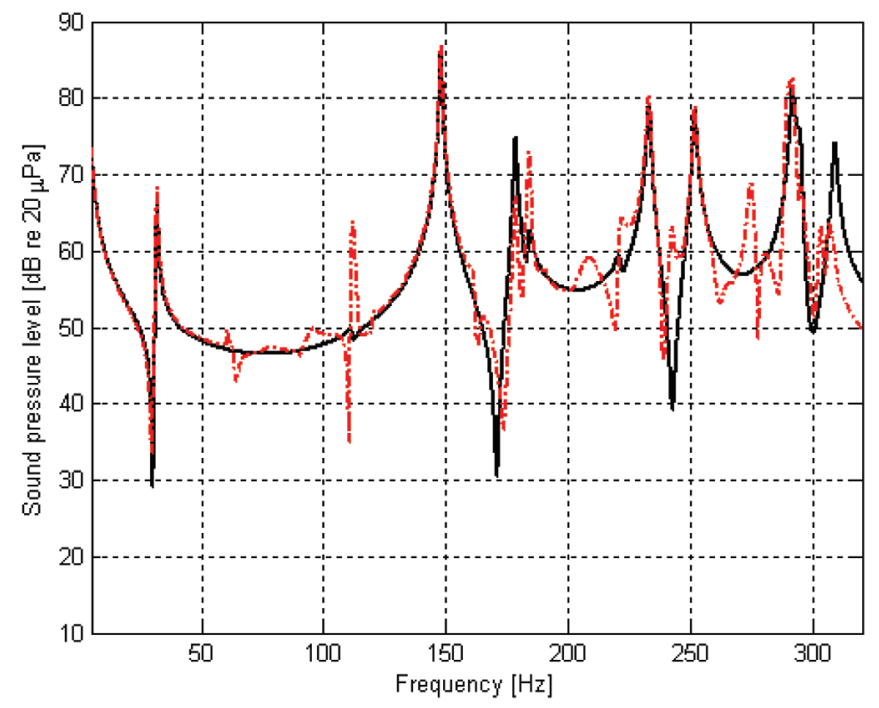

(b)

FIG. 8. (Color online) Secondary sound pressure frequency responses due to a $10^{-5} \mathrm{~m}^{3} / \mathrm{s} \mathrm{rms}$ deterministic acoustic volume velocity control input at $(0.200 \mathrm{~m}, 0.350 \mathrm{~m}, 0.140 \mathrm{~m})$ for (a) nominal case and (b) worst case. Solid line, the true sound pressure response; dash-dotted line, the estimated sound pressure response from the virtual sensor. $\square$, first and seventh panel-controlled modes; $\odot$, second-sixth cavity-controlled modes.

the control performance. In particular, when the control system is fully determined with as many secondary/control sources as the virtual sensors and $G_{\hat{v} u}$ is reasonably accurate and invertible, the control performance depends solely on the accuracy of the virtual sensor in estimating the primary sound field at the virtual location. In practice, the "inversion" of secondary transfer function can be implemented using adaptive filtering method such as using the filtered-x LMS algorithm (Elliott, 2001). In this work, to avoid excessive control input due to inaccurate virtual sensing secondary path, the magnitude of control input was limited to 0.8 of the magnitude of primary disturbance $d$.

Figure 9 shows the active control results for both nominal and worst cases. It is shown that even for the worst-case system, the controller performed reasonably well over the $300 \mathrm{~Hz}$ bandwidth, although there was an increase of noise 


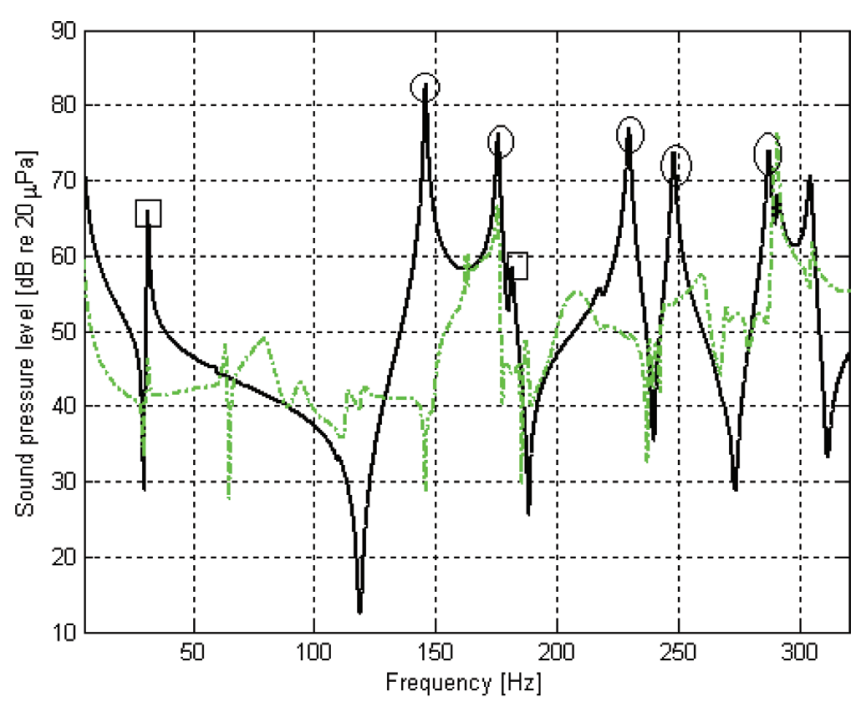

(a)

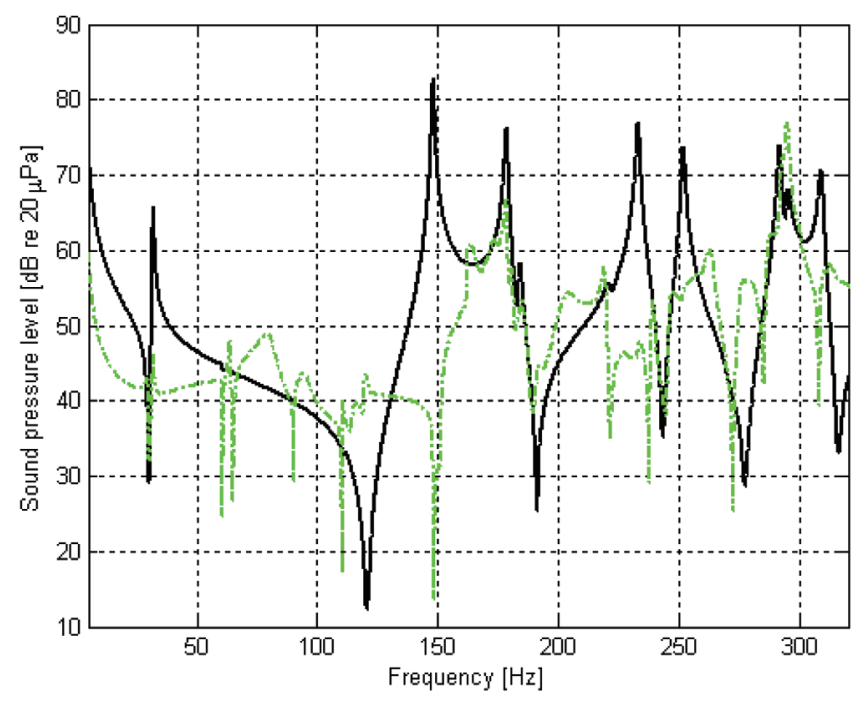

(b)

FIG. 9. (Color online) Active tonal noise control using virtual sensing for (a) nominal case and (b) worst case. Solid line, the primary sound pressure response; dash-dotted line, the controlled sound pressure response. $\square$, first and seventh panel-controlled modes; $\odot$, second-sixth cavity-controlled modes.

at off-resonance regions. Lower noise reductions were observed particularly at frequency range of 162-177 Hz due to relatively low secondary path responses (see Fig. 8) and limiting of maximum control input. It is interesting to see that there was a noise increase around the resonance of the seventh cavity-controlled mode at around 287-295 Hz, just next to the sixth cavity-controlled resonance. The poor control performance in this region was due to the fact that this mode was not prioritized in the structural sensor placement elaborated in Halim et al. (2001), leading to a low structural response as shown in Fig. 4(b). To improve the control performance, the structural sensor location can be modified to allow a higher resonance response for the mode, although possibly at the expense of other modes.

Finally, although the proposed robust virtual sensor design can readily be used with multiple structural sensors, the work was focused on using a single structural sensor only, as a performance benchmark, to demonstrate the accuracy of virtual sensor regardless of the number of sensors used. However, the sensing performance of the virtual sensor can further be improved by increasing the number of structural sensors. As mentioned by Halim et al. (2010), it is not generally possible to locate a structural sensor to achieve high modal observability levels for a large number of modes, and a compromise by prioritizing some important modes is thus required. The ability of structural sensor to observe certain modes can have a direct impact on the virtual sensor performance and robustness. In this case, multiple structural sensors can reduce the limitation of a single structural sensor. They can be strategically located on a flexible panel to achieve higher modal observability levels for a larger number of modes.

\section{CONCLUSIONS}

The work proposed a design methodology for developing a broadband robust virtual sensor for vibro-acoustic systems. In contrast to previously developed virtual sensors, structural sensors were used instead of acoustic sensors, allowing a compact set of structural sensors including micro-electro-mechanical (MEMS) or piezoelectric sensors to be used for sensing and active noise control applications. The virtual sensing robustness was explicitly considered to guarantee a certain sensing performance against dynamic uncertainties that were expected to occur in a practical vibro-acoustic system.

A practical virtual sensor design method was proposed for vibro-acoustic sensing and active control applications. Multiple Kalman sub-filters were used to construct the robust virtual sensor filter via the minimax optimization method. The basic design principle is to obtain Kalman sub-filters from the representative dynamic models and to optimize the convex combination of those sub-filters to achieve the best worst-case virtual sensing performance. This method can thus be readily extended for applications involving complex vibro-acoustic systems, whose uncertainty dynamic models are obtained from numerical modeling or system identification methods.

The numerical validation on a rectangular panel-cavity system demonstrated that it was possible to sense the interior sound pressure, particularly the one contributed by the cavity-controlled modes, using a structural sensor alone. The robustness of virtual sensing performance could be sufficiently achieved even for the worst-case dynamics. It was shown that it is important to judiciously place the structural sensor for obtaining effective virtual sensing performance. As expected, the active noise control performance was found to be dependent on virtual sensing accuracy. It was demonstrated that an effective noise control performance could still be achieved even at the worst-case dynamic. The proposed virtual sensor can thus be used for attractive active control applications for creating multiple zones of quiet by using a fixed set of structural sensors.

\section{ACKNOWLEDGMENTS}

The authors wish to acknowledge support given to them by the Research Grants Council of HKSAR through Grant No. PolyU 5132/07E. 
Bullmore, A. J., Nelson, P. A., Curtis, R. D., and Elliott, S. J. (1987). "The active minimization of harmonic enclosed sound fields, part II: A computer simulation," J. Sound Vib. 117, 15-33.

Cazzolato, B. S. (1999). "Sensing systems for active control of sound transmission in cavities," Ph.D. thesis, Adelaide University, South Australia, Chap. 5-7, pp. 221-318.

Cazzolato, B. S. (2002). "An adaptive LMS virtual microphone," in Proceedings of Active 2002, Southampton, UK, pp. 105-116.

Cheng, L. (1994). "Fluid structural coupling of a plate-ended cylindrical shell vibration and internal sound field," J. Sound Vib. 174(5), 641-654.

Elliott, S. J. (2001). Signal Processing for Active Control (Academic Press, San Diego, CA), Chaps. 2 and 4, pp. 49-102, 177-232.

Elliott, S. J., and David, A. (1992). "A virtual microphone arrangement for local active sound control," in Proceedings of the 1st International Conference on Motion and Vibration Control, Yokohama, Japan, pp. 1027-1031.

Elliott, S. J., and Garcia-Bonito, J. (1995). "Active cancellation of pressure and pressure gradient in a diffuse sound field," J. Sound Vib. 186(4), 696-704

Elliott, S. J., Joseph, P., Bullmore, A. J., and Nelson, P. A. (1988). "Active cancellation at a point in a pure tone diffuse sound field," J. Sound Vib. 120(1), 183-189.

Fahy, F. (1985). Sound and Structural Vibration: Radiation, Transmission and Response (Academic Press, London), Chap. 6, pp. 241-269.

Gelb, A. (Ed.) (1974). Applied Optimal Estimation (MIT, Cambridge, MA), Chap. 4, pp. 102-155.

Geromel, J. C., and Martins, R. C. D. (2009). "A new method to $\mathrm{H}_{2}$ robust filter design," Linear Algebra Appl. 430, 145-154.

Halim, D., Cheng, L., and Su, Z. (2010). "Robust virtual sensors for vibroacoustic applications using structural sensing," on CD-ROM in Proceedings of Inter-noise 2010 -39th International Congress on Noise Control Engineering: Noise and Sustainability, June 13-16, Lisbon, Portugal.

Hill, S. G., Tanaka, N., and Snyder, S. D. (2009). "A generalised approach for active control of structural-interior global noise," J. Sound Vib. 326(3-5), 456-475.

Kim, S. M., and Brennan, M. J. (2000). "Active control of harmonic sound transmission into an acoustic enclosure using both structural and acoustic actuators," J. Acoust. Soc. Am. 107(5), 2523-2534.

Lewis, F. L., Xie, L., and Popa, D. (2008). Optimal and Robust Estimation: With an Introduction to Stochastic Control Theory, 2nd ed. (CRC Press, Boca Raton, FL), Chaps. 2, 3, and 6, pp. 59-212, 315-351.

Li, D. S., and Cheng, L. (2010). "The design of synthesized structural acoustic sensors for active control of interior noise with experimental validation," J. Sound Vib. 329(2), 123-139.
Ljung, L. (1999). System Identification: Theory for the User, 2nd ed. (Prentice Hall, Upper Saddle River, NJ), Chap. 4, pp. 79-139.

Moreau, D., Cazzolato, B. S., Zander, A. C., and Petersen, C. D. (2008). "A review of virtual sensing algorithm for active noise control," Algorithms 1, 69-99.

Moreau, D., Ghan, J., Cazzolato, B. S., and Zander, A. C. (2009). "Active noise control in a pure tone diffuse sound field using virtual sensing," J. Acoust. Soc. Am. 125(6), 3742-3755.

Pan, J., and Bies, D. A. (1990). "The effect of fluid-structural coupling on sound waves in an enclosure-Theoretical part," J. Acoust. Soc. Am. 87(2), 692-707.

Pan, J., and Hansen, C. (1991). "Active control of noise transmission through a panel cavity: II. Experimental study," J. Acoust. Soc. Am. 90(3), 1488-1492.

Pawelczyk, M. (2003). "Multiple input-multiple output adaptive feedback control strategies for the active headrest system: Design and real-time implementation,” Int. J. Adapt. Control Signal Process. 17(10), 785-800.

Petersen, C. D., Fraanje, R., Cazzolato, B. S., Zander, A. C., and Hansen, C. H. (2008). "A Kalman filter approach to virtual sensing for active noise control," Mech. Syst. Signal Process. 22, 490-508.

Petersen, C., Zander, A., Cazzolato, B., and Hansen, C. (2007). "A moving zone of quiet for narrowband noise in a one-dimensional duct using virtual sensing," J. Acoust. Soc. Am. 121(3), 1459-1470.

Popovich, S. (1997). "Active acoustic control in remote regions," U.S. Patent No. 5, 701, 350.

Rafaely, B., and Elliott, S. J. (1999). " $\mathrm{H}_{2} / \mathrm{H}_{\infty}$ active control of sound in a headrest: Design and implementation," IEEE Trans. Control Syst. Technol. 7(1), 79-84.

Roure, A., and Albarrazin, A. (1999). "The remote microphone technique for active noise control," in Proceedings of Active 99, Fort Lauderdale, FL, pp. 1233-1244.

Snyder, S., and Tanaka, N. (1993). "On feed forward active control of sound and vibration using error signals," J. Acoust. Soc. Am. 94(4), 2181-2193.

Tanaka, N., and Kobayashi, K. (2006). "Cluster control of acoustic potential energy in a structural/acoustic cavity," J. Acoust. Soc. Am. 119(5), 2758-2771.

Thomas, D., Nelson, P., and Elliott, S. (1993). "Active control of the transmission of sound through a thin cylindrical shell. Part I: The minimisation of vibrational energy," J. Sound Vib. 167(1), 91-111.

Wenzel, T. A., Burnham, K. J., Blundell, M. V., and Williams, R. A. (2007). "Kalman filter as a virtual sensor: Applied to automotive stability system," Trans. Inst. Meas. Control (London) 29(2), 95-115. 